\title{
Review \\ CGRP Receptor Antagonists and 5-HT1F Receptor Agonist in the Treatment of Migraine
}

\author{
Matilde Capi ${ }^{1}$, Valerio De Angelis ${ }^{2}$, Donatella De Bernardini ${ }^{1}$, Ottavia De Luca ${ }^{3}$, Fabiola Cipolla ${ }^{2}$, \\ Luana Lionetto $^{1}$, Maurizio Simmaco ${ }^{1,3,4}$ and Paolo Martelletti ${ }^{2, *(D)}$ \\ 1 Laboratory of Clinical Biochemistry, Mass Spectrometry Unit, Sant'Andrea University Hospital, \\ 00185 Rome, Italy; matildecapi@gmail.com (M.C.); donatelladb@yahoo.it (D.D.B.); \\ luanalionetto@gmail.com (L.L.); maurizio.simmaco@uniroma1.it (M.S.) \\ 2 Department of Clinical and Molecular Medicine, Sapienza University of Rome, 00185 Rome, Italy; \\ valerio.deangelis@uniroma1.it (V.D.A.); fabiola.cipolla@alice.it (F.C.) \\ 3 Laboratory of Clinical Biochemistry, Advanced Molecular Diagnostic Unit, Sant'Andrea University Hospital, \\ 00185 Rome, Italy; ottavia_deluca@yahoo.it \\ 4 Department of Neurosciences, Mental Health and Sensory Organs (NESMOS), Sapienza University, \\ 00185 Rome, Italy \\ * Correspondence: paolo.martelletti@uniroma1.it
}

check for updates

Citation: Capi, M.; De Angelis, V.; De Bernardini, D.; De Luca, O.;

Cipolla, F.; Lionetto, L.; Simmaco, M.; Martelletti, P. CGRP Receptor Antagonists and 5-HT1F Receptor Agonist in the Treatment of Migraine. J. Clin. Med. 2021, 10, 1429. http:// doi.org/10.3390/jcm10071429

Academic Editors: Anna P. Andreou and Tissa Wijeratne

Received: 21 December 2020

Accepted: 20 March 2021

Published: 1 April 2021

Publisher's Note: MDPI stays neutral with regard to jurisdictional claims in published maps and institutional affiliations.

Copyright: (c) 2021 by the authors. Licensee MDPI, Basel, Switzerland. This article is an open access article distributed under the terms and conditions of the Creative Commons Attribution (CC BY) license (https:// creativecommons.org/licenses/by/ $4.0 /)$.

\begin{abstract}
Discovering that calcitonin-related peptide (CGRP) plays a key role in the complex pathophysiology of migraine has allowed us to make great strides in the development of new approaches for acute and preventive treatment. This evidence has led to the development of small molecules antagonist molecules of the CGRP receptor ("gepants") and of a new class of medications called "Ditans". This review presents the data from clinical trials reporting the efficacy, safety, and tolerability of the new drugs used in the treatment of migraines. Evidences show that therapeutic approaches targeted to CGRP have the potential to transform the clinical management of migraine, even though its appropriate place has yet to be determined with accuracy.
\end{abstract}

Keywords: migraine; CGRP; therapy; gepants; ditans; antagonism; CGRP receptor

\section{Introduction}

Migraine is not a symptom, but a complex neurological disease which, according to the World Health Organization, represents the third most frequent disease under the age of 50. A study carried out in 2016 by the Global Burden of Disease (GBD) classifies migraine as the sixth most widespread and one of the main causes of disability worldwide, as it often manifests in the working ages, in young adults and in women of childbearing age [1]. It represents, therefore, a real social problem, in terms of years of life lived with a disability, which especially afflicts individuals aged 15 to 49 . Migraine symptoms include pain, sensitivity to light, sound, odors, and changes in vision, in addition to nausea, vomiting, tingling, and speech disorders, and have significant disabling effects on the physical, social, and professional functioning of the patient [2]. Since the first drug for the treatment of migraine was introduced, other medications including calcium channel antagonists, antidepressants, antiepileptics, and antihypertensives developed for indications other than migraine, have entered the field based on clinical studies. However, efficacy is often insufficient, and the incidence of adverse effects leads to the suspension of the therapeutic treatment [3]. The discovery of the key role of the peptide related to the gene of calcitonin (CGRP) in the complex pathophysiology of the migraine has made it possible to make large progress in developing new approaches to preventive and acute treatment [4]. This has led to the development of small molecules antagonist of the CGRP receptor ("gepants") and monoclonal antibodies (mAbs) affecting the CGRP or its receptor. In studies on monoclonal antibody therapies, no serious safety or tolerability problems have been identified. These monoclonal antibodies are the first specific treatment for the prevention of migraine, 
and they have good efficacy and tolerability [5]. In addition, thanks to the long half-life, their allow treatment schemes that are well accepted by the patient, with one administration every 4 or 12 weeks [6]. All monoclonal antibodies acting on the CGRP have been shown to modify and improve quality of life (Qol) and headache-related disability. Erenumab, Galcanezumab, and Fremanezumab have been approved both by the US Food and Drug Administration (FDA) and the European Medicine Agency (EMA) for migraine treatment; the latest (Eptinezumab) was approved by the FDA in February 2020. Another valid support in the pharmacological treatment of migraine has been given by the development of the gepants.

Gepants, selective antagonists of the CGRP receptor, are small molecules that compete with endogenous CGRP at a specific receptors level. Some years ago, first generation gepants like olcegepant, telcagepant, BI44370TA, and MK-3207 proved to have good efficacy but their development was discouraged because of a scarcely favorable safety profile and the route of administration, only intravenous [7]. The search for new gepants thus focused on molecules that did not present chemical structures potentially related to hepatotoxicity problems. Studies led to the development of two new candidates, rimegepant and ubrogepant, recently approved by the FDA for the treatment of acute migraine headache, while atogepant remains an experimental drug for preventive therapy. Vazegepant, with positive preliminary data, would be the first intranasal gepant for acute treatment. Further studies are needed in order to define a safety and efficacy and tolerability profile for new pharmacological treatments but the new therapies available up to date consent to approach the precision medicine for migraine, allowing specific therapies for individual patients. Recently new molecules called "Ditans" have been developed; they are selective agonist for 5-HT1F receptor with a better tolerability profile compared to triptans, due to their very low affinity for 5-HT1B-1D receptors subtypes, known for their involvement in vasoconstrictive mechanisms such as Lasmiditan [8].

\section{Methods}

This literature review research was carried out in November 2020 on MEDLINE (www.ncbi.nlm.nih.gov / pubmed, last accessed on 20 March 2021) for both original and review papers on the mechanism of action of CGRP antagonists and 5-HT1F agonists in migraine, and for randomized double-blind placebo-controlled trials on the new generation of Gepants and Ditans until 2020. For the purpose of this review, we also performed research on www.clinicaltrials.gov and on The International Register of Clinical Trials Portal (www.who.int/ictrp, last accessed on 30 November 2020) for named Gepants and Ditans. We considered only outcomes from randomized controlled trials (RCTs) of adult subjects affected with migraine with or without aura and studies describing the pharmacological treatment of migraine using CGRP Receptor Antagonists and the 5-HT1F receptor agonist. We excluded studies not regarding migraine or involving people under 18 years, studies having an unclearly defined design, target population, and/or results, all the open label studies or trials that did not have placebo controls, and all the studies in healthy subjects. We limited our search to articles regarding human subjects and published in English.

\section{Molecular Mechanisms of CGRP Antagonists in Migraine}

Many studies have hypothesized the key role played by the CGRP in the pathophysiology of migraine, confirmed over the years by clinical findings even if its specific pathogenic mechanisms in migraine is still being investigated [9]. In these studies, CGRP is found to be released from trigeminal ganglia neurons, its transcription increases under conditions imitating neurogenic inflammation, and migraine pharmacotherapies can both inhibit CGRP transcription and reduce CGRP release, and tumor necrosis factor- $\alpha$ (TNF- $\alpha$ ), an endogenous inflammatory mediator involved in migraine, can arouse CGRP transcription [9]. CGRP is a neuropeptide consisting of 37 amino acids and belongs to a family of peptides that includes calcitonin (CT), adrenomedullin (AM), and amyl (AMY). This peptide presents itself in $\alpha$ and $\beta$ isoforms, produced by two different genes (respectively CALC I 
and CALC II) located in two different sites of the same chromosome: chromosome 11 [10]. These two isoforms, in human, differ by three amino acids and perform similar biological activities. The $\alpha$ isoform is considered the main neuronal form, being located at the level of sensory nerve endings of the central and peripheral nervous system; $\beta$-CGRP was initially found in the enteric nervous system, while recent evidence shows that $\beta$ isoform may be released together with $\alpha$-CGRP at the vascular level [11]. The CGRP receptor, related to a protein $G$, is formed by three subunits: receptor activity-modifying protein 1 (RAMP1), calcitonin-like receptor (CLR), and the component protein receptor (CPR) [12]. The mechanism by which CGRP binds to the receptor can be interpreted according to the general model of the two domains indicated by Hoare. In this model, the C-terminal of the peptide ligand firstly binds with high affinity to the extracellular portion N-terminal receptor forming a so-called affinity trap [13]. This initial event increases the local peptide concentration, which then allows for the N-terminal portion of the CGRP to interact with the juxtamembrane region of the CLR, thus activating the receptor and determining the accumulation of cyclic adenosine monophosphate (Camp) [14]. The activation of CGRP receptors regulates the increase of intracellular levels of cAMP and cyclic guanosine monophosphate (cGMP) in numerous body cells and these are the second messengers associated with vasodilation. The role of CGRP in vasodilation of peripheral vascular beds can be described through many vascular mechanisms. To date, the CGRP, when it binds to its receptor, induces a vasodilation through an endothelium-dependent and endothelium-independent mechanism [15]. In ats and humans' arteries level the CGRP, in its independent endothelium path, binds directly to the CGRP receptors on smooth muscle cells activating adenylate cyclase. This process produces an increase in cAMP levels resulting in vascular relaxation. CGRP also has the ability to stimulate the production of $\mathrm{NO}$ by acting via a receptor located on the endothelium (pathway dependent endothelium). In this way, the CGRP binds to its receptor and causes an increase in cAMP through adenylate cyclase. This process determines the activation of the enzyme NO synthetase (NOS) which leads to an increase in nitrogen oxide levels (NO). Then, NO activates the route of guanylate cyclase on smooth muscle cells leading to the production of cGMP and vascular relaxation [16]. The first person to recognize the implication of GCRP in migraine was Edvinsson in 1990 when he demonstrated its role in the cerebrovascular trigeminal neuron system as vasoactive constituent and therefore implied in the cerebral vasoconstriction processes [17]. The recognition of the important role of the CGRP in the pathogenesis of migraine has aroused great interest in the study of new therapeutic approaches. The CGRP receptor antagonists have shown a good clinical efficacy in the treatment of acute migraine attacks and offer a valid alternative to current therapies.

\section{Preventative New Gepants}

Preventive treatment therapy aims to lower the frequency, intensity, and duration of migraine headaches increasing the efficacy of drugs and reducing the risk of worsening and the chronicization of the headache. It also aims to reduce symptomatic drugs assumption. Preventive treatment is efficacious when at least one of the following conditions occurs: a $50 \%$ reduction in the frequency of migraine days, a significant reduction in migraine attacks or their severity or an increased response to therapeutic treatment, a reduction in disability due to the disease, or an improvement in Qol [18]. Preventative treatment drugs vary from antiepileptics to calcium channel blockers or angiotensin converting enzyme inhibitors. It is very important to choose the right treatment for each individual, depending on the efficacy and side-effect profile and the patient's comorbidities or preferences [19]. Seven gepants have been developed since 2004. Gepants are small molecule calcitonin gene-related peptide (CGRP) receptor antagonists and will be prescribed for a number of patients who found other therapies ineffective or with present cardiac or cerebrovascular risks factors. 


\subsection{Atogepant}

4.1.1. Efficacy

Atogepant, a gepant discovered by Merck Sharp \& Dohme, was the first oral CGRP antagonist developed for the preventative treatment of migraine. To date, A Phase 2/3, Multicenter, Randomized, Double-Blind, Placebo Controlled, Parallel-Group Study was conducted to evaluate efficacy, safety, and tolerability of multiple dosing regimens of oral AGN-241689 in episodic migraine prevention (NCT02848326) [20]. 834 participants with migraines, with or without aura, were given once daily (QD) atogepant $10 \mathrm{mg}(n=93)$, $30 \mathrm{mg}(n=183) 60 \mathrm{mg}(n=186)$ and twice daily (BID) $30 \mathrm{mg}(n=86)$ and $60 \mathrm{mg}(n=91)$ respect to placebo group $(n=186)$. The mean of monthly migraine days (MMD) at baseline was $7.67 \pm 2.49$. Regarding the efficacy, results showed a reduction from baseline in MMD on average compared to the placebo group. Particularly, the MMD change was -2.85 (placebo group), -4.0 (10 mg QD, $p=0.024),-3.76$ (30 mg QD, $p=0.039),-3.55$ (60 mg QD, $p=0.039),-4.23$ (30 mg BID, $p=0.003)$ and $-4.14(60 \mathrm{mg}$ BID, $p=0.003)$. A further analysis of the data showed that the least squares mean was -2.93 for placebo and -4.31 (10 $\mathrm{mg}$ QD), -4.17 (30 mg QD), -4.23 (30 mg BID), -3.86 (60 mg QD) and -4.32 (60 mg BID). A secondary endpoint was the proportion of patients who achieved at least a $50 \%$ reduction in mean monthly migraine days. The data reported a reduction of $40.4 \%$ (placebo group), 57.6\% (atogepant $10 \mathrm{mg}$ QD), 53.3\% (atogepant $30 \mathrm{mg}$ QD), 58.2\% (atogepant $60 \mathrm{mg}$ QD), $52.0 \%$ (atogepant $30 \mathrm{mg} \mathrm{BID)}$ and $62.1 \%$ (atogepant $60 \mathrm{mg}$ QD). The change of least squares mean was -2.42 (placebo group), -3.71(10 mg QD), -3.86 (30 mg QD), -3.53(60 mg QD) it -3.77 (30 $\mathrm{mg}$ BID) it was -3.64 (60 mg BID).

\subsubsection{Safety and Tolerability}

In a phase $2 / 3$, multicenter, randomized, double-blind, placebo controlled, parallelgroup study (NCT02848326) assessed the safety profile of atogepant. The most adverse events (AEs) were nausea, constipation, nasopharyngitis, and urinary tract infection (frequency $\geq 5 \%$ ) in all groups compared to placebo. Alanine transaminase or aspartate aminotransferase were found $>3 \times$ the upper limit normal (ULN) in $1.7 \%$ (placebo group), $2.2 \%$ (10 mg QD), $0.6 \%$ (30 mg QD), 1.7\% (60 QD), 1.2\% (30 mg BID) and 1.1\% (60 mg BID). The results indicate that atogepant is well tolerated and drug-related serious AEs were absent [21].

\section{Acute New Gepants}

The acute treatment of migraine attacks provides numerous options for choosing the drug to use, but unfortunately still none of them is proven to be effective, in equal measure, for all migraine patients. Therapy must be personalized and cannot predict which type of acute therapy will be effective for a specific patient and therefore it is of-ten necessary to try with various types of molecules before finding the most suitable one. A drug therapy effectiveness determines an improvement of Qol, greater adherence to therapy and treatment in general for its part as well as reducing the risk of chronicization and abuse of the symptomatic drugs [22]. The two main categories of drugs with evidence of efficacy in the acute treatment of migraine attack remain today the triptans and the nonsteroidal anti-inflammatory drugs (NSAIDs). Up to date, FDA has approved many drugs for migraine acute treatment in adults, but their efficacy is not homogeneous and their use can also be limited by safety concerns [23].

\subsection{Ubrogepant}

5.1.1. Efficacy

Ubrogepant (MK-1602) is a novel oral CGRP receptor antagonist, and it has been developed for the acute treatment of migraine. Its absorption in humans is rapid and its apparent half-life is about three $h$. Its metabolization pathway goes through the liver, principally via CYP3A4, and it is also a $p$-glycoprotein substrate [24]. Two randomized, double-blind, placebo-controlled trials [<ACHIEVE I (NCT02828020) and ACHIEVE II 
(NCT02867709)] were carried out in order to assess the efficacy of UBRELVY for the acute treatment of migraine.

In ACHIEVE I, ubrogepant $50 \mathrm{mg}, 100 \mathrm{mg}$ or placebo were administered to 1327 patients. Pain freedom at $2 \mathrm{~h}$ was $19.2 \%$ (vs. placebo, $p=0.0023$ ) in the $50 \mathrm{mg}$ group, $21.2 \%$ (vs. placebo, $p=0.0003$ ) in the $100 \mathrm{mg}$ group and $11.8 \%$ in the placebo group, while freedom from most bothersome symptom (MBS) at 2-h was reported by $38.6 \%$ in the $50 \mathrm{mg}$ group (vs. placebo, $p=0.0023$ ) and $37.7 \%$ in the $100 \mathrm{mg}$ group (vs. placebo, $p=0.0023$ ) compared to $27.8 \%$ for placebo, respectively [25].

The ACHIEVE II trial was carried out on 1686 patients administered respectively with ubrogepant $25 \mathrm{mg}$, ubrogepant $50 \mathrm{mg}$ or placebo. Pain freedom at $2 \mathrm{~h}$ was $20.7 \%$ (vs. placebo, $p=0.0285$ ) in the $25 \mathrm{mg}$ group, $21.8 \%$ (vs. placebo, $p=0.0129$ ) in the $50 \mathrm{mg}$ group and $14.3 \%$ in the placebo group, while freedom from MBS at 2-h was reported by $34.1 \%$ of the $25 \mathrm{mg}$ group (vs. placebo, $p=0.0711$ ) and $38.9 \%$ of the $50 \mathrm{mg}$ group (vs. placebo, $p=0.0129$ ), compared to $27.4 \%$ for placebo. The $25 \mathrm{mg}$ dose was not statistically significant compared to placebo [26].

Another multicenter, randomized, open-label extension study, UBR-MD-04, included 1254 patients who completed one between ACHIEVE I (NCT02828020) or ACHIEVE II (NCT02867709) trials. These patients were administered with both $50 \mathrm{mg}$ and $100 \mathrm{mg}$ doses and they could take a second dose or a rescue medication if they did not respond to the first dose or if they experienced a migraine recurrence. The effects of ubrogepant were observed in the short long-term ACHIEVE clinical trial (NCT02873221). These studies were aimed to assess the long-term safety and tolerability of discontinuous treatment with ubrogepant for the acute treatment of migraine (with or without aura) over 1 year. 1254 subjects were enrolled to evaluate the efficacy of $50 \mathrm{mg}(n=417)$ and $100 \mathrm{mg}(n=420)$ dose of ubrogepant respect to a standard therapy prescribed by the physician $(n=417)$ [27]. Results after one year reported pain freedom at $2 \mathrm{~h}$ after the initial dose in $\approx 24 \%$ of ubrogepant-treated (50 or $100 \mathrm{mg}$ ) attacks and pain relief at $2 \mathrm{~h}$ post-dose in $\approx 67 \%$ of ubrogepant-treated attacks [28].

A phase Illb, multicenter, randomized, double-blind, placebo-controlled trial (NCT01613248) was conducted to investigate the efficacy and safety of ubrogepant [29]. In this study, 834 subjects with migraine disease were enrolled and randomized to ubrogepant $1 \mathrm{mg}(n=138), 10 \mathrm{mg}$ $(n=139), 25 \mathrm{mg}(n=139), 50 \mathrm{mg}(n=139), 100 \mathrm{mg}(n=140)$ respect to placebo group $(n=139)$. The percentual of Reporting Pain Freedom (PF) at $2 \mathrm{~h}$ post-dose was 5.6 (1 mg dose), 14.8\% (10 mg), 21.4\% (25 mg), 21\% (50 mg), 25.5\% (100 mg) respect to 8.9\% (placebo). The percentage of participants reporting pain relief (PR) at $2 \mathrm{~h}$ post-dose was $37.4 \%$ (1 mg dose), $52.8 \%$ (10 mg), $53.4 \%$ (25 mg), 57.1\% (50 mg), 58.8\% (100 mg) respect to $44.6 \%$ (placebo). The percentage of Participant Reporting Absence of Photophobia at $2 \mathrm{~h}$ post-dose was 37.4\% (1 mg dose), 43.5\% (10 mg), 39.8\% (25 mg), 47.6\% (50 mg), 54.9\% (100 mg) respect to 30.4\% (placebo). Significant differences respect to placebo group were also seen for ubrogepant $50 \mathrm{mg}$ regarding to absence of phonophobia and photophobia at $2 \mathrm{~h}$, sustained pain freedom and sustained pain relief 2-24 and $2-48 \mathrm{~h}$, total migraine freedom at $2 \mathrm{~h}$ and at a $2-24 \mathrm{~h}$. Significant data were found for ubrogepant $25 \mathrm{mg}$ regarding a sustained pain freedom $2-24 \mathrm{~h}$ and $2-48 \mathrm{~h}$, for sustained pain relief $2-48 \mathrm{~h}$ and for total migraine freedom at $2 \mathrm{~h}$ [30].

\subsubsection{Safety and Tolerability}

In the ACHIEVE I and II studies, the AEs most commonly reported were nausea, somnolence, and dry mouth with a frequency lower than 5\% (ACHIEVE I) and lower than $2.5 \%$, (ACHIEVE II). Hepatotoxicity was not reported $[25,26]$.

In a randomized, open-label extension study (NCT02873221), the treatment related AEs were reported by the $10.4 \%$ and $10.5 \%$ of the ubrogepant $50 \mathrm{mg}$ and $100 \mathrm{mg}$ groups, respectively. One patient in the ubrogepant $50 \mathrm{mg}$ group, with a previous history of supraventricular tachycardia with ablation, experienced a serious AEs (sinus tachycardia) that the investigator considered treatment related. The results also reported 20 cases of AST / ALT levels $\geq 3 \times$ ULN in the two groups, and two of these cases in the 50 mg group 
were considered treatment related, while one in the $100 \mathrm{mg}$ group was considered only probably related to the presence of confounding factors [28].

In a phase $\mathrm{Ilb}$, multicenter, randomized, double-blind, placebo-controlled trial (NCT01613248) the reporting absence of nausea at $2 \mathrm{~h}$ post-dose was $59.8 \%$ ( $1 \mathrm{mg}$ dose), $67.6 \%$ (10 mg), 73.8\% $(25 \mathrm{mg}), 68.6 \%(50 \mathrm{mg}), 70.6 \%$ (100 mg) respect to $62.5 \%$ (placebo). The incidences of AEs in the first $48 \mathrm{~h}$ after administration were similar for the ubrogepant groups and were mostly dry mouth, nausea, fatigue, dizziness, and somnolence that did not appear to be dose dependent. There were no changes in liver enzymes or laboratory values. An increase of AST $>3$ occurred in the $50 \mathrm{mg}$ group but this event was declared unrelated to the treatment [30].

\subsection{Zavegepant}

\subsubsection{Efficacy}

Biohaven Pharmaceuticals developed Zavegepant (synonyms BHV-3500), a calcitonin gene-related peptide (CGRP) receptor antagonist, for the treatment of migraine. It is administered intranasally and is currently undergoing clinical trials. A phase 2 and 3 multicenter, randomized, double-blind, placebo controlled clinical trial (NCT03872453) was recently carried out on safety and efficacy of three different intranasal dose levels of BHV-3500 for the acute treatment of migraine. Patients enrolled $(n=2154)$ have had from 2 to 8 moderate to severe migraines per month during the previous 3 months, suffering from migraine for more than 1 year with an onset prior to 50 years of age. They received BHV-3500 $5 \mathrm{mg}, 10 \mathrm{mg}$ and $20 \mathrm{mg}$ matching placebo. Pain relief at $2 \mathrm{~h}$ was respectively $19.6 \%(p$-value $=0.1214), 22.5 \%(p$-value $=0.0113)$ and $23.1 \%(p$-value $=0.0055)$ compared to placebo group $(n=401,15.5 \%)$ for the groups treated with vazegepant $5 \mathrm{mg}(n=387)$, $10 \mathrm{mg}(n=391)$ and $20 \mathrm{mg}(n=402)$ [31].

Treatment with BHV-3500 showed a freedom from MBS in 39.0\% subjects treated with $5 \mathrm{mg}$ ( $p$-value: 0.1162$), 41.9 \%$ in subjects treated with $10 \mathrm{mg}$ ( $p$-value: 0.0155$)$ and $42.5 \%$ in subjects treated with $20 \mathrm{mg}$ ( $p$-value: 0.0094$)$, respectively, compared to placebo (33.7\%) [32].

\subsubsection{Safety and Tolerability}

To date, there are no available data on tolerability. The pre-clinical data of Intranasal vazegepant reported by Biohaven showed a good tolerability in the single dose trial. The AEs in more than $5 \%$ of the treated subjects were dysgeusia (13.5 to $16.1 \%$ vazegepant, $3.5 \%$ placebo) and nasal discomfort (1.3 to $5.2 \%$ vazegepant, $0.2 \%$ placebo), the majority of them $(>80 \%)$ being of mild intensity. Data on the patients treated with $5 \mathrm{mg}(n=388), 10 \mathrm{mg}$ $(n=394), 20 \mathrm{mg}(n=403)$ and placebo group $(n=403)$ reported no signal of hepatoxicity as no AST or ALT $>3 \times \mathrm{ULN}$, or total bilirubin $>2 \times \mathrm{ULN}$ [32].

\subsection{Rimegepant}

\subsubsection{Efficacy}

Rimegepant is a calcitonin-related peptide receptor antagonist which was found to be effective in the treatment of acute migraine in its oral administration, especially in those patients whose symptoms do not respond to triptans. Unlike triptans, rimegepant has no vasoconstrictor effects and is not contraindicated in patients with cardiovascular disease. Efficacy, safety and tolerability have been demonstrated by several clinical studies. A Phase 2 and 3 studies (NCT03235479 and NCT03266588) recruited 1485 subjects and 3019 subjects respectively [33,34]. In the phase 3 study, $75 \mathrm{mg}$ of rimegepant dose were administered to patients in respect to a placebo group. The primary outcomes have been analyzed were freedom from pain at $2 \mathrm{~h}$ post-dose, freedom from MBS at $2 \mathrm{~h}$ post-dose while the secondary outcomes included a several parameter such as photo-phobia-free, pain relief, nausea-free, rescue medications sustained pain free and pain re-lapse at $2 \mathrm{~h}$ or 24-48 h. The results showed a significant and durable clinical effect with a single dose of rimegepant in pain freedom, freedom from MBS, pain relief and recovery of normal function and other outcome measures. 
A phase $\mathrm{Ilb}$, double-blind, randomized, placebo controlled, dose-ranging trial (NCT01430442) evaluate the efficacy of rimegepant (BMS-927711) compared with placebo in the acute treatment of migraine. A group of 1026 participants received one dose of rimegepant (among the $10 \mathrm{mg}$, $25 \mathrm{mg}, 75 \mathrm{mg}$, $150 \mathrm{mg}$, $300 \mathrm{mg}$, or $600 \mathrm{mg}$ ) or placebo or sumatriptan (100 mg). More patients in the rimegepant $75 \mathrm{mg}(31.4 \%, p=0.002), 150 \mathrm{mg}(32.9 \%, p<0.001)$, and $300 \mathrm{mg}(29.7 \%, p=0.002)$ groups and the sumatriptan group $(35 \%, p<0.001)$ reached freedom from pain at two $h$ post dose vs. placebo $(15.3 \%)$, and this result was significant. The secondary endpoint, sustained pain freedom from two to $24 \mathrm{~h}$ post-dose, was significantly reached from rimegepant doses (25-600 mg) compared to placebo [35].

A phase 3 (NCT03237845) evaluates the efficacy of rimegepant compared with placebo in migraine acute treatment. 1499 participants were enrolled to receive to rimegepant $75 \mathrm{mg}$ oral tablet, or placebo $75 \mathrm{mg}$ oral tablet. All the primary endpoints were met, and most patients achieved pain relief, the benefit was durable ( 24 and $48 \mathrm{~h}$ ), achieved normal function and used a lower number of rescue meds [36].

A double-blind, randomized, placebo-controlled, multicenter phase 3 clinical trial (NCT03461757) evaluated the efficacy of rimegepant in subjects with Acute Migraines respect to placebo group. 1811 participants were enrolled to receive $75 \mathrm{mg}$ of Rimegepant dose respect to placebo. The results showed that rimegepant orally disintegrating tablet was superior to placebo at $2 \mathrm{~h}$ post-dose in freedom from pain $(21 \%$ vs. $11 \%, p<0.0001)$ and freedom from the MBS (35\% vs. $27 \%, p=0.0009)$ [37].

\subsubsection{Safety and Tolerability}

Regarding AEs, phase 2 and 3 clinical trials (NCT03235479 and NCT03266588) showed that rimegepant $75 \mathrm{mg}$ oral tablet proved to be tolerable, safe and comparable to placebo on tests of liver function. The majority of AEs reported in NCT03266588 were of mild or moderate in intensity, being the most common upper respiratory tract infection $(8.5 \%)$, nasopharyngitis $(6.4 \%)$, and sinusitis $(4.8 \%)[33,34]$.

In a Phase IIb, double-blind, randomized, placebo controlled, dose-ranging trial (NCT01430442), the most common AE observed in the rimegepant dosing groups was nausea, which was found to be dose dependent: $1.4 \%$ in the $10 \mathrm{mg}$; 0 in the $25 \mathrm{mg} ; 3 \%$ in each of the $75 \mathrm{mg}$ and $150 \mathrm{mg}$ dose groups; $4 \%$ in the $300 \mathrm{mg}$; and $8 \%$ in the $600 \mathrm{mg}$ group [35].

A randomized, phase 3 clinical trial (NCT03237845) reported that the common AEs in $75 \mathrm{mg}$ rimegepant dose were nausea $(1.8 \%)$, urinary tract infection (1.5\%). Safety and tolerability profiles were similar to placebo and better if compared to the past triptan experience [36].

In a randomized controlled trial (NCT03461757) no serious AEs were reported. The most common were nausea ( $2 \%$ in rimegepant vs. $1 \%$ in placebo group) and urinary tract infection ( $1 \%$ in rimegepant dose vs. $1 \%$ in placebo group). Two participants, one for each group, reported abnormal transaminase concentration of more than $3 \times$ the upper limit and it was not related to study medication. Moreover, no elevations in bilirubin greater than 2 times the upper limit of normal were reported [37].

\section{5-HT1D Agonists-Ditans}

Numerous studies on the pathophysiology of migraine have shown the crucial importance of the serotoninergic system in the genesis of the attack that, and this has led to the development of 5-HT1 receptor agonists, including 5-HT1B/D and 5-HT1F, in order to achieve a better treatment for this burdensome disease.

\subsection{Molecular Mechanisms of 5-HT1F Receptor Agonist}

When the serotoninergic receptor 5HT1B-1D is activated it induces vasoconstriction, neurogenic inflammation blockage, and a reduction of cephalalgic symptoms. These effects produce a risk for patients with cardiovascular and/or cerebrovascular diseases [38]. Recently, new molecules have been developed called "Ditans". These molecules are 
selective agonist for 5-HT1F receptor and have a low affinity with the 5-HT1B-1D receptors subtypes, giving them a better tolerability profile compared to triptans with a lower involvement in vasoconstrictive mechanisms [39]. It is assumed that the activation of the 5HT1F receptor inhibits trigeminal impulses hyperpolarizing nerve terminals, but not many studies have investigated its downstream pathways. Among the 5-HT1F receptor agonists being developed, only one molecule entered a clinical trial so far: LY334370, a molecule with a 100-fold higher selectivity for the 5-HT1F receptor than the 5-HT1B and 5-HT1D receptors [40].

\subsection{Lasmiditan \\ 6.2.1. Efficacy}

For acute migraine therapy with or without aura, the FDA approved the drug Lasmiditan (previously known as COL-144 and LY573144) under the trade name Reyvow (Eli Lilly, Indianapolis, Indiana, USA) and is available in tablets of $50 \mathrm{mg}$ and $100 \mathrm{mg}$. This drug has a different chemical structure from other compounds and is part of a new class of drugs called "Ditans". Lasmiditan is a new receptor agonist 5-HT1F and the activation of 5-HT1F receptors decreases the expression of c-Fos, a neural activation marker, in the caudate nucleus of the trigeminum, without having any vascular effects [41]. Two randomized, double-blind, placebo-controlled trials [SAMURAI (NCT02439320) and SPARTAN (NCT02605174)] were con-ducted to evaluate the efficacy of lasmiditan in the acute treatment of migraine, enrolling patients with a migraine history according to the criteria of the International Classification of Headache Disorders (ICHD-II) [42,43]. The primary objective of each trial was to evaluate the efficacy of lasmiditan vs placebo in treating migraine-related headache pain and MBS. SAMURAI randomized patients to lasmiditan $100 \mathrm{mg}(n=744)$, or $200 \mathrm{mg}(n=745)$ or placebo $(n=742)$ [26]. The patients recruited for the two trials were enlisted in the open GLADIATOR (NCT02565186) study, conducted up to 12 months. These clinical trial randomized patients to lasmiditan $100 \mathrm{mg}(n=1046)$, or $200 \mathrm{mg}(n=1125)$ or placebo $(n=2171)$ in order to assess safety, efficacy and tolerability of long-term intermittent use of lasmiditan $100 \mathrm{mg}$ and $200 \mathrm{mg}$ for the acute treatment of migraine [44]. Its long-term intermittent use for the acute treatment of migraine [44]. In the SAMURAI clinical trial, pain freedom at $2 \mathrm{~h}$ was observed in $28.2 \%$ (vs. placebo, $p<0.001$ ) in the $100 \mathrm{mg}$ group, $32.2 \%$ (vs. placebo, $p<0.001$ ) in the $200 \mathrm{mg}$ group and $15.3 \%$ in the placebo group. Freedom from most bothersome symptom at $2 \mathrm{~h}$ was $40.9 \%$ (vs. placebo, $p<0.001$ ) in the $100 \mathrm{mg}$ group, $40.7 \%$ (vs. placebo, $p<0.001$ ) in the $200 \mathrm{mg}$ group compared to $29.5 \%$ in the placebo group. The most common adverse events were dizziness and paresthesia, both from mild to moderate intensity. The SPARTAN clinical trial showed similar data, proving a good efficacy also for the $50 \mathrm{mg}$ group: pain freedom at $2 \mathrm{~h}$ was observed in $28.6 \%$ (vs. placebo, $p=0.003$ ) in the $50 \mathrm{mg}$ group, $31.4 \%$ (vs. placebo, $p<0.001$ ) in the $100 \mathrm{mg}$ group, $38.8 \%$ (vs. placebo, $p<0.001$ ) in the $200 \mathrm{mg}$ group and $21.3 \%$ in placebo group. Freedom from most bothersome symptoms at $2 \mathrm{~h}$ occurred in $40.8 \%$ (vs. placebo, $p=0.009$ ) in the $50 \mathrm{mg}$ group, $44.2 \%$ (vs. placebo, $p<0.001$ ) in the $100 \mathrm{mg}$ group, $48.7 \%$ (vs. placebo, $p<0.001$ ) in the $200 \mathrm{mg}$ group and $33.5 \%$ in the placebo group. Adverse events reported were dizziness, paresthesia, somnolence, fatigue, nausea and lethargy.

A recent phase 2 clinical trial (NCT00883051) evaluate the safety and efficacy of treating migraine headache of a series of oral doses of COL-144, in order to select a dose or doses for further evaluation. These clinical trial randomized patients to lasmiditan $50 \mathrm{mg}(106), 100 \mathrm{mg}(n=104), 200 \mathrm{mg}(n=100), 400 \mathrm{mg}(n=99)$ and placebo $(n=103)$. Lasmiditan 50,100, 200, or $400 \mathrm{mg}$ doses were significantly superior to placebo in reducing migraine pain intensity, and this was especially true for lasmiditan $200 \mathrm{mg}(19 \%, p=0.032)$ and $400 \mathrm{mg}(28 \%, p=0.0007)[45,46]$. All the efficacy results reported in this review are summarized in Table 1. 
Table 1. Gepants and ditans efficacy data.

\begin{tabular}{|c|c|c|c|c|c|c|c|c|c|}
\hline \multirow[t]{2}{*}{ Drug } & \multirow[t]{2}{*}{ Identifier } & \multirow[t]{2}{*}{ Intervention } & $\begin{array}{c}\text { Monthly Migraine } \\
\text { Days }\end{array}$ & \multirow{2}{*}{$\begin{array}{l}50 \% \text { Reduction in } \\
\text { Mean MMD }\end{array}$} & \multirow{2}{*}{$\begin{array}{l}\text { Least-Squares } \\
\text { Means }\end{array}$} & \multirow[t]{2}{*}{ Pain Free at $2 h$} & \multirow[t]{2}{*}{$\begin{array}{c}\text { Bothersome } \\
\text { Symptoms (MBS) }\end{array}$} & \multirow[t]{2}{*}{ Pain Relief at $2 \mathrm{~h}$} & \multirow[t]{2}{*}{ Reference } \\
\hline & & & (MMD) & & & & & & \\
\hline \multirow{5}{*}{ Atogepant } & \multirow{5}{*}{ NCT02848326 } & $10 \mathrm{mg}$ QD & -4.00 (10 mg QD) & $57.6 \%(10 \mathrm{mg} Q D)$ & -3.71 (10 mg QD) & \multirow{5}{*}{ Not available } & \multirow{5}{*}{ Not available } & \multirow{5}{*}{ Not available } & \multirow{5}{*}{ [20] } \\
\hline & & $30 \mathrm{mg}$ QD & -3.76 (30 mg QD) & $53.3 \%$ (30 mg QD) & $-3.86(30 \mathrm{mg}$ QD) & & & & \\
\hline & & $30 \mathrm{mg}$ BID & $-4.23(30 \mathrm{mg}$ BID & $52.0 \%(30 \mathrm{mg}$ BID $)$ & -3.77 (30 mg BID) & & & & \\
\hline & & $60 \mathrm{mg}$ QD & -3.55 (60 mg QD) & $58.2 \%(60 \mathrm{mg}$ QD) & $-3.53(60 \mathrm{mg}$ QD) & & & & \\
\hline & & $60 \mathrm{mg}$ BID & $-4.14(60 \mathrm{mg}$ BID $)$ & $62.1 \%(60 \mathrm{mg}$ BID $)$ & $-3.64(60 \mathrm{mg}$ BID $)$ & & & & \\
\hline \multirow{10}{*}{ Ubrogepant } & \multirow{2}{*}{ NCT02828020 } & $50 \mathrm{mg}$ & \multirow{2}{*}{ Not available } & \multirow{2}{*}{ Not available } & \multirow{2}{*}{ Not available } & $19.2 \%(50 \mathrm{mg})$ & $38.6 \%(50 \mathrm{mg})$ & \multirow{2}{*}{ Not available } & \multirow{2}{*}{ [25] } \\
\hline & & $100 \mathrm{mg}$ & & & & $21.2 \%$ (100 mg) & $37.7 \%$ (100 mg) & & \\
\hline & \multirow{2}{*}{ NCT02867709 } & $25 \mathrm{mg}$ & \multirow{2}{*}{ Not available } & \multirow{2}{*}{ Not available } & \multirow{2}{*}{ Not available } & $20.7 \%$ (25 mg) & $34.1 \%(25 \mathrm{mg})$ & \multirow{2}{*}{ Not available } & \multirow{2}{*}{ [26] } \\
\hline & & $50 \mathrm{mg}$ & & & & $21.8 \%(50 \mathrm{mg})$ & $38.9 \%(50 \mathrm{mg})$ & & \\
\hline & \multirow{2}{*}{ NCT02873221 } & $50 \mathrm{mg}$ & \multirow{2}{*}{ Not available } & \multirow{2}{*}{ Not available } & \multirow{2}{*}{ Not available } & $24 \%$ (50 and 100 & \multirow{2}{*}{ Not available } & \multirow{2}{*}{ Not available } & [27] \\
\hline & & $100 \mathrm{mg}$ & & & & mg) & & & \\
\hline & NCT01613248 & $10 \mathrm{mg}$ & Not available & Not available & Not available & $14.8 \%(10 \mathrm{mg})$ & Not available & $52.8 \%(10 \mathrm{mg})$ & [29] \\
\hline & & $25 \mathrm{mg}$ & & & & $21.4 \%(25 \mathrm{mg})$ & & $53.4 \%(25 \mathrm{mg})$ & \\
\hline & & $50 \mathrm{mg}$ & & & & $21.0 \%(50 \mathrm{mg})$ & & $57.1 \%(50 \mathrm{mg})$ & \\
\hline & & $100 \mathrm{mg}$ & & & & $25.5 \%$ (100 mg) & & $58.8 \%(100 \mathrm{mg})$ & \\
\hline & & $10 \mathrm{mg}$ & & & & $22.5 \%(10 \mathrm{mg})$ & $41.9 \%(10 \mathrm{mg})$ & & \\
\hline Zavegepant & NCT03872453 & $20 \mathrm{mg}$ & Not available & Not available & Not available & $23.1 \%(20 \mathrm{mg})$ & $42.5 \%(20 \mathrm{mg})$ & Not available & [31] \\
\hline & & $5 \mathrm{mg}$ & & & & $19.6 \%(5 \mathrm{mg})$ & $39.0 \%$ (5 mg) & & \\
\hline
\end{tabular}


Table 1. Cont.

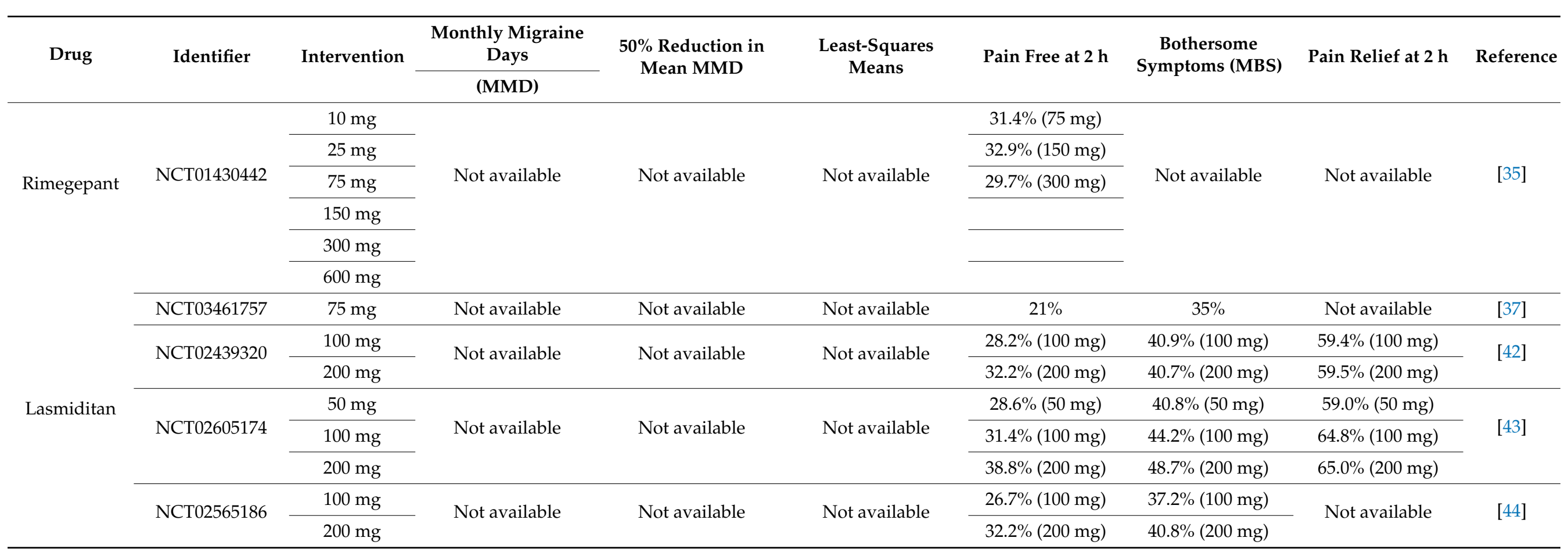




\subsubsection{Safety and Tolerability}

In both these studies (NCT02439320 and NCT02605174) lasmiditan proved its safety and no serious AEs were reported [26].

In an open-label, long-term, safety study of Lasmiditan (NCT02565186), the most frequent treatment-emergent adverse events (TEAEs) $(>2 \%)$ reported were dizziness $(18.6 \%)$, somnolence (sleepiness or drowsiness; $8.5 \%$ ), paresthesia (tingling or numb sensation on the skin; $6.8 \%$ ), fatigue (5.5\%), nausea $(4.7 \%)$ and asthenia (physical weakness or lack of energy; $2.0 \%$ ) [47].

In a double blind, randomized, placebo-controlled, parallel group dose-ranging study of oral COL-144 in the Acute Treatment of Migraine (NCT00883051), the AEs reported were dizziness, fatigue, vertigo, paresthesia, and somnolence [48]. All the clinical studies analyzed in this review are summarized in Table 2. 
Table 2. Gepants and Ditans studies analyzed.

\begin{tabular}{|c|c|c|c|c|c|c|c|c|}
\hline Drug & Identifier & Disease & $\begin{array}{l}\text { Estimated } \\
\text { Enrollment }\end{array}$ & Study Description & Intervention & Phase & Sponsors & Reference \\
\hline \multirow{5}{*}{ Atogepant } & \multirow{5}{*}{ NCT02848326 } & \multirow{5}{*}{$\begin{array}{l}\text { Migraine with or } \\
\text { without aura }\end{array}$} & \multirow{5}{*}{834} & \multirow{5}{*}{$\begin{array}{l}\text { Safety and tolerability of } \\
\text { Atogepant. }\end{array}$} & $10 \mathrm{mg}$ QD & \multirow{5}{*}{$2 / 3$} & \multirow{5}{*}{ Allergan } & \multirow{5}{*}{ [20] } \\
\hline & & & & & $30 \mathrm{mg}$ QD & & & \\
\hline & & & & & $30 \mathrm{mg}$ BID & & & \\
\hline & & & & & $60 \mathrm{mg}$ QD & & & \\
\hline & & & & & $60 \mathrm{mg}$ BID & & & \\
\hline \multirow{9}{*}{ Ubrogepant } & NCT02828020 & $\begin{array}{l}\text { Migraine with or } \\
\text { without aura }\end{array}$ & 1672 & $\begin{array}{c}\text { Efficacy, safety, and tolerability of } 2 \\
\text { doses of ubrogepant. }\end{array}$ & $100 \mathrm{mg}$ & 3 & Allergan & [25] \\
\hline & \multirow{2}{*}{ NCT02867709 } & \multirow{2}{*}{$\begin{array}{l}\text { Migraine with or } \\
\text { without aura }\end{array}$} & \multirow{2}{*}{1686} & \multirow{2}{*}{$\begin{array}{l}\text { Efficacy, safety, and tolerability of } 2 \\
\text { doses of ubrogepant. }\end{array}$} & $25 \mathrm{mg}$ & \multirow{2}{*}{3} & \multirow{2}{*}{ Allergan } & \multirow{2}{*}{ [26] } \\
\hline & & & & & $50 \mathrm{mg}$ & & & \\
\hline & \multirow{2}{*}{ NCT02873221 } & \multirow{2}{*}{$\begin{array}{l}\text { Migraine with or } \\
\text { without aura }\end{array}$} & \multirow{2}{*}{1254} & \multirow{2}{*}{$\begin{array}{c}\text { Evaluate the long-term safety and } \\
\text { tolerability of ubrogepant over } 1 \\
\text { year. }\end{array}$} & $50 \mathrm{mg}$ & \multirow{2}{*}{3} & \multirow{2}{*}{ Allergan } & \multirow{2}{*}{ [27] } \\
\hline & & & & & $100 \mathrm{mg}$ & & & \\
\hline & \multirow{4}{*}{ NCT01613248 } & \multirow{4}{*}{ Migraine } & \multirow{4}{*}{834} & \multirow{4}{*}{$\begin{array}{l}\text { Assess the effectiveness, safety and } \\
\text { tolerability of a range of doses of } \\
\text { MK-1602 versus placebo. }\end{array}$} & $1 \mathrm{mg}$ & \multirow{4}{*}{2} & & \\
\hline & & & & & $25 \mathrm{mg}$ & & Hilergant & {$[29]$} \\
\hline & & & & & $50 \mathrm{mg}$ & & & \\
\hline & & & & & $100 \mathrm{mg}$ & & & \\
\hline & & & & & $10 \mathrm{mg}$ & & & \\
\hline Zavegepant & NCT03872453 & Migraine & 2154 & different intranasal dose levels. & $20 \mathrm{mg}$ & $2 / 3$ & Pharmaceuticals & [31] \\
\hline & & & & & $5 \mathrm{mg}$ & & & \\
\hline
\end{tabular}


Table 2. Cont.

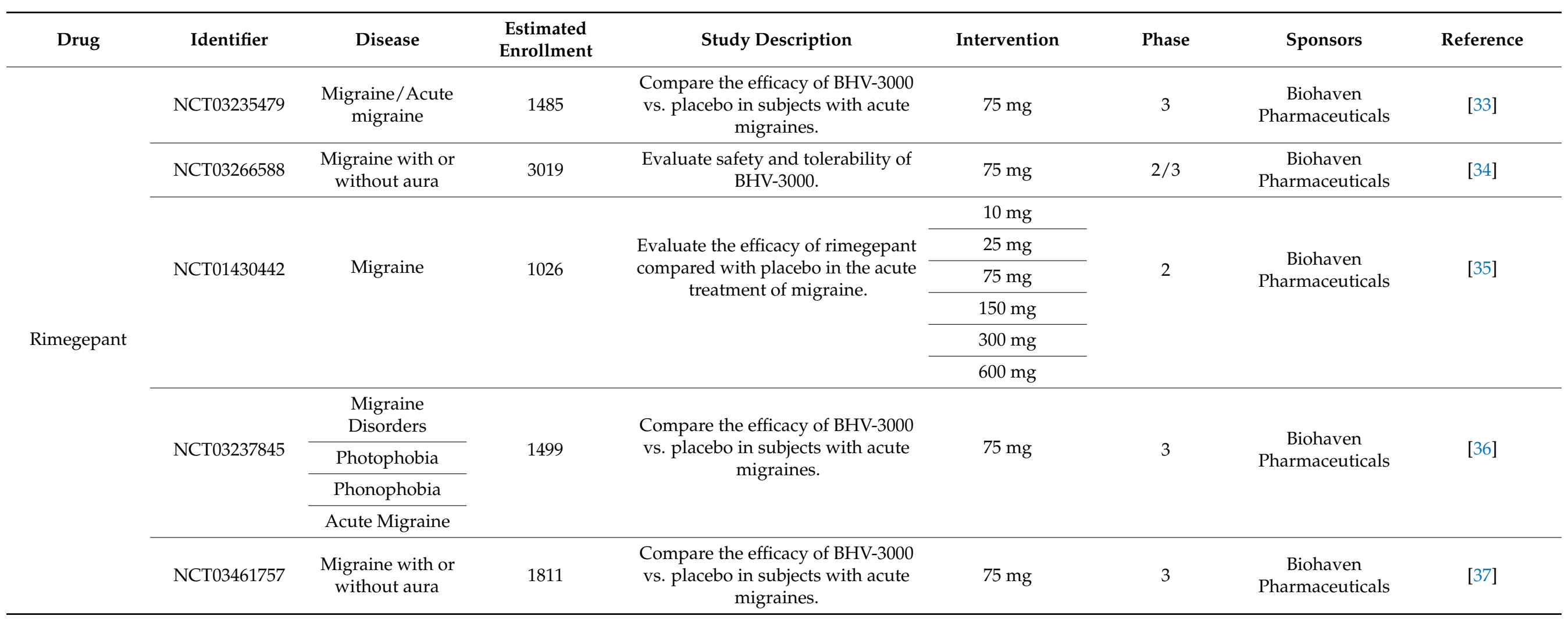


Table 2. Cont.

\begin{tabular}{|c|c|c|c|c|c|c|c|c|}
\hline Drug & Identifier & Disease & $\begin{array}{l}\text { Estimated } \\
\text { Enrollment }\end{array}$ & Study Description & Intervention & Phase & Sponsors & Reference \\
\hline \multirow{11}{*}{ Lasmiditan } & \multirow[t]{2}{*}{ NCT02439320 } & \multirow[t]{2}{*}{ Acute Migraine } & \multirow[t]{2}{*}{2231} & \multirow{2}{*}{$\begin{array}{l}\text { A prospective study in participants } \\
\text { with disabling migraine. }\end{array}$} & $100 \mathrm{mg}$ & \multirow[t]{2}{*}{3} & $\begin{array}{l}\text { Eli Lilly and } \\
\text { Company }\end{array}$ & \multirow[t]{2}{*}[42]{} \\
\hline & & & & & $200 \mathrm{mg}$ & & $\begin{array}{c}\text { CoLucid } \\
\text { Pharmaceuticals }\end{array}$ & \\
\hline & \multirow{3}{*}{ NCT02605174 } & \multirow{3}{*}{$\begin{array}{l}\text { Migraine with or } \\
\text { without Aura }\end{array}$} & \multirow{3}{*}{3005} & \multirow{3}{*}{$\begin{array}{l}\text { A prospective study in participants } \\
\text { with disabling migraine. }\end{array}$} & $50 \mathrm{mg}$ & \multirow{3}{*}{3} & $\begin{array}{l}\text { Eli Lilly and } \\
\text { Company }\end{array}$ & \multirow{3}{*}{ [43] } \\
\hline & & & & & $100 \mathrm{mg}$ & & $\begin{array}{c}\text { CoLucid } \\
\text { Pharmaceuticals }\end{array}$ & \\
\hline & & & & & $200 \mathrm{mg}$ & & & \\
\hline & \multirow[t]{2}{*}{ NCT02565186 } & \multirow{2}{*}{$\begin{array}{l}\text { Migraine } \\
\text { Disorders }\end{array}$} & \multirow[t]{2}{*}{2171} & \multirow{2}{*}{$\begin{array}{l}\text { Safety Study of Lasmiditan in } \\
\text { the acute treatment of migraine. }\end{array}$} & $100 \mathrm{mg}$ & \multirow[t]{2}{*}{3} & $\begin{array}{l}\text { Eli Lilly and } \\
\text { Company }\end{array}$ & \multirow[t]{2}{*}{ [44] } \\
\hline & & & & & $200 \mathrm{mg}$ & & $\begin{array}{c}\text { CoLucid } \\
\text { Pharmaceuticals }\end{array}$ & \\
\hline & \multirow{4}{*}{ NCT00883051 } & \multirow{4}{*}{$\begin{array}{l}\text { Migraine } \\
\text { disorders }\end{array}$} & \multirow{4}{*}{512} & $\begin{array}{l}\text { Efficacy and safety of a range of } \\
\text { oral doses of }\end{array}$ & $50 \mathrm{mg}$ & \multirow{4}{*}{2} & $\begin{array}{l}\text { Eli Lilly and } \\
\text { Company }\end{array}$ & \multirow{4}{*}[46]{} \\
\hline & & & & $\begin{array}{l}\text { COL-144 in treating migraine } \\
\text { headache. }\end{array}$ & $100 \mathrm{mg}$ & & $\begin{array}{c}\text { CoLucid } \\
\text { Pharmaceuticals }\end{array}$ & \\
\hline & & & & & $200 \mathrm{mg}$ & & & \\
\hline & & & & & $400 \mathrm{mg}$ & & & \\
\hline
\end{tabular}




\section{Discussion}

The pharmacological treatments currently available for migraine headaches are able to provide complete pain relief and, sustained within $2 \mathrm{~h}$, only in a small part of patients. Very often, the excessive use of these medicines is associated with the onset of headaches from abuse or overuse medication overuse. To date, the ineffectiveness of the pharmacological treatments is also impeded by the discontinuity due to the poor tolerability of some drugs, or the contraindications for the presence of cardiovascular disorders or significant risks. In recent years, clinical and preclinical research has largely treated the pathophysiological basis of migraine, and calcitonin-gene related peptide (CGRP) seems to play a crucial role in migraine attacks. The CGRP was initially de-scribed as a powerful mediator able to perform different functions in the nervous system, intestine and heart. Subsequently, his role as a vasodilator was discovered at the level of the cerebral and peripheral circulation. Therefore, CGRP is a valid therapeutic target and "Gepants" development represents a great opportunity for the anti-migraine therapies field. Gepants, selective CGRP receptor antagonists, are small molecules that compete with endogenous CGRP at the level of specific receptors. The gepants of first generation, olcegepant, telcagepant, BI44370TA and MK-3207, had shown a few years ago a good effectiveness but their development had been discouraged by an unfavorable safety profile or the need for its intravenous route of administration. In recent years, further clinical studies have been carried out on new molecules at different therapeutic doses and all the gepants appear to have similar effectiveness, minimal side effects, and to be safe. CGRP receptor antagonists are the first non-serotoninergic drugs specific for migraine without a vasoconstrictive action. Therefore, they could be indicated in patients with vascular diseases as peripheral vascular disease and coronary artery disease. Another pharmacological class in clinical trials is the ditans, serotoninergic agonists with specific affinity towards 5-HT1F receptors, are another pharmacological class investigated in clinical trials. Among the ditans, the molecule currently under study for the acute treatment of migraine is lasmiditan, that showed good efficacy and tolerability in two randomized and controlled studies (intravenous and oral administration) and was approved by the FDA in October 2019.

\section{Conclusions}

The new pharmacological treatments available today for the acute or preventive treatment of migraine are a huge step forward in the management of the disease. In particular, the options for a specific treatment for migraine are now strongly increased since the introduction of many new approaches to the peptide related to the CGRP receptors. In this sense, especially the introduction of specific monoclonal antibodies for migraine has represented a big step forward as well as the development of the new molecules acting as antagonists for the CGRP receptors. In the near future, the introduction on large scale of the CGRP agonists will provide a better tool for migraine therapies. The introduction of specific preventive therapies for migraine headaches based on sound science is an encouraging development for patients, in addition to the possibility of a significantly improved quality of life for those who respond to new treatments. These new therapies will allow to get a step closer to precision medicine for migraine, allowing a specific therapy for any individual patient. This could definitely improve the care of all migraine patients worldwide.

Author Contributions: M.C. was the principal investigator and corresponding author responsible for manuscript design, original draft writing, literature search, review and interpretation, manuscript writing, and manuscript revision; V.D.A. helped with manuscript writing and review, D.D.B. helped with manuscript writing and review; O.D.L. helped with manuscript preparation; F.C. was responsible for collecting funds supervision and L.L.; M.S. and P.M. were responsible and supervision the work. All authors have read and agreed to the published version of the manuscript.

Funding: This research received no external funding. 
Data Availability Statement: All the data sources are available within the article, have been properly cited and are publicly accessible.

Conflicts of Interest: The authors declare no conflict of interest.

\section{References}

1. GBD 2016 Headache Collaborators. Global, regional, and national burden of migraine and tension-type headache, 1990-2016: A systematic analysis for the Global Burden of Disease Study 2016. Lancet Neurol. 2018, 17, 954-976. [CrossRef]

2. Giamberardino, M.A.; Affaitati, G.; Costantini, R.; Cipollone, F.; Martelletti, P. Calcitonin gene-related peptide receptor as a novel target for the management of people with episodic migraine: Current evidence and safety profile of erenumab. J. Pain Res. 2017, 10, 2751-2760. [CrossRef]

3. Giamberardino, M.A.; Affaitati, G.; Curto, M.; Negro, A.; Costantini, R.; Martelletti, P. Anti-CGRP monoclonal antibodies in migraine: Current perspectives. Intern. Emerg. Med. 2016, 11, 1045-1057. [CrossRef] [PubMed]

4. Deen, M.; Correnti, E.; Kamm, K.; Kelderman, T.; Papetti, L.; Rubio-Beltrán, E.; Vigneri, S.; Edvinsson, L.; Maassen Van Den Brink, A.; European Headache Federation School of Advanced Studies (EHF-SAS). Blocking CGRP in migraine patients-A review of pros and cons. J. Headache Pain 2017, 18, 96. [CrossRef] [PubMed]

5. Martelletti, P. The Application of CGRP(r) Monoclonal Antibodies in Migraine Spectrum: Needs and Priorities. BioDrugs 2017, 31, 483-485. [CrossRef] [PubMed]

6. Agostoni, E.C.; Barbanti, P.; Calabresi, P.; Colombo, B.; Cortelli, P.; Frediani, F.; Geppetti, P.; Grazzi, L.; Leone, M.; Martelletti, P.; et al. Current and emerging evidence-based treatment options in chronic migraine: A narrative review. J. Headache Pain 2019, 20, 92. [CrossRef]

7. Moreno-Ajona, D.; Pérez-Rodríguez, A.; Goadsby, P.J. Gepants, calcitonin-gene-related peptide receptor antagonists: What could be their role in migraine treatment? Curr. Opin. Neurol. 2020, 33, 309-315. [CrossRef]

8. Curto, M.; Cipolla, F.; Cisale, G.Y.; Capi, M.; Spuntarelli, V.; Guglielmetti, M.; Martelletti, P.; Lionetto, L. Profiling lasmiditan as a treatment option for migraine. Expert Opin. Pharmacother. 2020, 21, 147-153. [CrossRef]

9. Durham, P.L. Calcitonin gene-related peptide (CGRP) and migraine. Headache 2006, 46 (Suppl. S1), S3-S8. [CrossRef] [PubMed]

10. Smillie, S.J.; Brain, S.D. Calcitonin gene-related peptide (CGRP) and its role in hypertension. Neuropeptides 2011, 45, 93-104. [CrossRef] [PubMed]

11. Tringali, G.; Navarra, P. Anti-CGRP and anti-CGRP receptor monoclonal antibodies as antimigraine agents. Potential differences in safety profile postulated on a pathophysiological basis. Peptides 2019, 116, 16-21. [CrossRef]

12. Lionetto, L.; Curto, M.; Cisale, G.Y.; Capi, M.; Cipolla, F.; Guglielmetti, M.; Martelletti, P. Fremanezumab for the preventive treatment of migraine in adults. Expert Rev. Clin. Pharmacol. 2019, 12, 741-748. [CrossRef]

13. Hoare, S.R. Mechanisms of peptide and nonpeptide ligand binding to Class B G-protein-coupled receptors. Drug Discov. Today 2005, 10, 417-427. [CrossRef]

14. Hargreaves, R.; Olesen, J. Calcitonin Gene-Related Peptide Modulators-The History and Renaissance of a New Migraine Drug Class. Headache 2019, 59, 951-970. [CrossRef]

15. Villalón, C.M.; Olesen, J. The role of CGRP in the pathophysiology of migraine and efficacy of CGRP receptor antagonists as acute antimigraine drugs. Pharmacol. Ther. 2009, 124, 309-323. [CrossRef] [PubMed]

16. Favoni, V.; Giani, L.; Al-Hassany, L.; Asioli, G.M.; Butera, C.; de Boer, I.; Guglielmetti, M.; Koniari, C.; Mavridis, T.; Vaikjärv, M.; et al. CGRP and migraine from a cardiovascular point of view: What do we expect from blocking CGRP? J. Headache Pain 2019, 20, 27. [CrossRef] [PubMed]

17. McCulloch, J.; Uddman, R.; Kingman, T.A.; Edvinsson, L. Calcitonin gene-related peptide: Functional role in cerebrovascular regulation. Proc. Natl. Acad. Sci. USA 1986, 83, 5731-5735. [CrossRef] [PubMed]

18. Silberstein, S.D. Preventive Migraine Treatment. Continuum 2015, 21, 973-989.

19. Lambru, G.; Andreou, A.P.; Guglielmetti, M.; Martelletti, P. Emerging drugs for migraine treatment: An update. Expert Opin. Emerg. Drugs 2018, 23, 301-318. [CrossRef] [PubMed]

20. Efficacy, Safety, and Tolerability of Multiple Dosing Regimens of Oral Atogepant (AGN-241689) in Episodic Migraine Prevention, NCT02848326. Available online: https: / clinicaltrials.gov/ct2/show / NCT02848326? cond=NCT02848326\&draw =2\&rank=1 (accessed on 22 November 2020).

21. Martelletti, P.; Cipolla, F.; Capi, M.; Curto, M.; Lionetto, L. Calcitonin gene-related peptide (CGRP) receptor antagonist, Preventive treatment of migraine. Drugs Future 2020, 45, 285. [CrossRef]

22. Pomes, L.M.; Guglielmetti, M.; Bertamino, E.; Simmaco, M.; Borro, M.; Martelletti, P. Optimising migraine treatment: From drug-drug interactions to personalized medicine. J. Headache Pain 2019, 20, 56. [CrossRef] [PubMed]

23. Center for Drug Evaluation and Research. Summary Review on Ubrogepant. Available online: https://www.accessdata.fda.gov / drugsatfda_docs/nda/2019/2117650rig1s000SumR.pdf (accessed on 22 November 2020).

24. Curto, M.; Capi, M.; Cipolla, F.; Cisale, G.Y.; Martelletti, P.; Lionetto, L. Ubrogepant for the treatment of migraine. Expert Opin. Pharmacother. 2020, 21, 755-759. [CrossRef] [PubMed]

25. Efficacy, Safety, and Tolerability Study of Oral Ubrogepant in the Acute Treatment of Migraine (ACHIEVE I), NCT02828020. Available online: https:// clinicaltrials.gov/ct2/show/NCT02828020?cond=NCT02828020\&draw=2\&rank=1 (accessed on 8 January 2021). 
26. Do, T.P.; Guo, S.; Ashina, M. Therapeutic novelties in migraine: New drugs, new hope? J. Headache Pain 2019, 20, 1-13. [CrossRef]

27. An Extension Study to Evaluate the Long-Term Safety and Tolerability of Ubrogepant in the Treatment of Migraine, NCT02873221. Available online: https: / / clinicaltrials.gov/ct2/ show / study / NCT02873221? cond=NCT02873221\&draw =2\&rank=1 (accessed on 8 January 2021).

28. Scott, L.J. Ubrogepant: First Approval. Drugs 2020, 80, 323-328. [CrossRef]

29. A Dose-Finding Study of MK-1602 in the Treatment of Acute Migraine (MK-1602-006), NCT01613248. Available online: https: / / clinicaltrials.gov / ct2/ show / NCT01613248? cond=NCT01613248\&draw=2\&rank=1 (accessed on 22 November 2020).

30. Voss, T.; Lipton, R.B.; Dodick, D.W.; Dupre, N.; Ge, J.Y.; Bachman, R.; Assaid, C.; Aurora, S.K.; Michelson, D. A phase IIb randomized, double-blind, placebo-controlled trial of ubrogepant for the acute treatment of migraine. Cephalalgia 2016, 36, 887898. [CrossRef]

31. Acute Treatment Trial in Adult Subjects with Migraines, NCT03872453. Available online: https://clinicaltrials.gov/ct2/show / NCT03872453? cond=NCT03872453\&draw=2\&rank=1 (accessed on 8 January 2021).

32. Biohaven Achieves Positive Topline Results in Pivotal Phase 2/3 Study of Vagezepant, the First and Only Intranasal CGRP Receptor Antagonist in Clinical Development for the Acute Treatment of Migraine. Available online: https://www.biohavenpharma. com/investors/news-events/press-releases/12-17-2019 (accessed on 8 January 2021).

33. Safety and Efficacy Study in Adult Subjects with Acute Migraines, NCT03235479. Available online: https://clinicaltrials.gov/ct2 / show / NCT03235479?cond=NCT03235479\&draw=2\&rank=1 (accessed on 8 January 2021).

34. Open Label Safety Study in Acute Treatment of Migraine, NCT03266588. Available online: https://clinicaltrials.gov/ct2/show / NCT03266588? cond=NCT03266588\&draw=2\&rank=1 (accessed on 8 January 2021).

35. Dose Ranging Study of Rimegepant (BMS-927711) for the Acute Treatment of Migraine, NCT01430442. Available online: https: / / clinicaltrials.gov/ct2/show / NCT01430442?cond=NCT01430442\&draw=2\&rank=1 (accessed on 8 January 2021).

36. Safety and Efficacy in Adult Subjects with Acute Migraines, NCT03237845. Available online: https://clinicaltrials.gov/ct2/show/ NCT03237845? cond=NCT03237845\&draw=2\&rank=1 (accessed on 8 January 2021).

37. Trial in Adult Subjects with Acute Migraines, NCT03461757. Available online: https://clinicaltrials.gov/ct2/show/NCT0346175 7 ? cond $=$ NCT03461757\&draw $=2 \&$ rank=1 (accessed on 8 January 2021).

38. Vila-Pueyo, M. Targeted 5-HT $1 \mathrm{~F}$ Therapies for Migraine. Neurotherapeutics 2018, 15, 291-303. [CrossRef]

39. Capi, M.; de Andrés, F.; Lionetto, L.; Gentile, G.; Cipolla, F.; Negro, A.; Borro, M.; Martelletti, P.; Curto, M. Lasmiditan for the treatment of migraine. Expert Opin. Investig. Drugs 2017, 26, 227-234. [CrossRef]

40. Neeb, L.; Meents, J.; Reuter, U. 5-HT(1F) Receptor agonists: A new treatment option for migraine attacks? Neurotherapeutics 2010, 7, 176-182. [CrossRef]

41. Oswald, J.C.; Schuster, N.M. Lasmiditan for the treatment of acute migraine: A review and potential role in clinical practice. J. Pain Res. 2018, 11, 2221-2227. [CrossRef]

42. Lasmiditan Compared to Placebo in the Acute Treatment of Migraine: (SAMURAI), NCT02439320. Available online: https: / / clinicaltrials.gov / ct2/ show / NCT02439320?cond=NCT02439320\&draw=2\&rank=1 (accessed on 24 March 2021).

43. Three Doses of Lasmiditan (50 mg, $100 \mathrm{mg}$ and $200 \mathrm{mg}$ ) Compared to Placebo in the Acute Treatment of Migraine (SPARTAN), NCT02605174. Available online: https: / clinicaltrials.gov/ct2/show / NCT02605174? cond=NCT02605174\&draw=2\&rank=1 (accessed on 8 January 2021).

44. An Open-Label, Long-Term, Safety Study of Lasmiditan for the Acute Treatment of Migraine (GLADIATOR), NCT02565186. Available online: https://clinicaltrials.gov/ct2/show / NCT02565186? cond=NCT02565186\&draw=2\&rank=1 (accessed on 8 January 2021).

45. Clemow, D.B.; Johnson, K.W.; Hochstetler, H.M.; Ossipov, M.H.; Hake, A.M.; Blumenfeld, A.M. Lasmiditan mechanism of action-Review of a selective 5-HT1F agonist. J. Headache Pain 2020, 21, 1-13. [CrossRef] [PubMed]

46. Dose-ranging Study of Oral COL-144 in Acute Migraine Treatment, NCT00883051. Available online: https: / clinicaltrials.gov / ct2/ show / NCT00883051?cond=NCT00883051\&draw=2\&rank=1 (accessed on 8 January 2021).

47. AAN 2019: Two New Analyses of Lasmiditan Phase 3 Studies Measured Onset of Effect and the Effect of Lasmiditan in Patients with Prior Triptan Experience. Available online: https:/ /investor.lilly.com/news-releases/news-release-details/aan-2019-twonew-analyses-lasmiditan-phase-3-studies-measured (accessed on 24 March 2021).

48. Färkkilä, M.; Diener, H.C.; Géraud, G.; Láinez, M.; Schoenen, J.; Harner, N.; Pilgrim, A.; Reuter, U.; COL MIG-202 Study Group. Efficacy and tolerability of lasmiditan, an oral 5-HT(1F) receptor agonist, for the acute treatment of migraine: A phase 2 randomised, placebo-controlled, parallel-group, dose-ranging study. Lancet Neurol. 2012, 11, 405-413. [CrossRef] 